\section{Géneros}

Multidisciplinary Journal of Gender Studies

\section{Hipatia Press \\ www.hipatiapress.com}

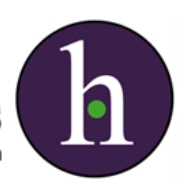

Instructions for authors, subscriptions and further details:

http://generos.hipatiapress.com

\title{
Aprendizaje con TIC para la Inclusión Digital. Las Mujeres como Tejedoras de las Redes Sociales.
}

María José Cabanillas Sánchez ${ }^{1}$

1) Universidad de Extremadura. España

Date of publication: June $25^{\text {th }}, 2017$

Edition period: June 2016 - October 2017

To cite this article: Cabanillas Sánchez, M.J. (2017). Aprendizaje con TIC para la Inclusión Digital. Las Mujeres como Tejedoras de las Redes Sociales [Review of the book]. Multidisciplinary Journal of Gender Studies, 6(2), 1385-1388. doi: 10.4471/generos.2017.2667

To link this article: http://dx.doi.org/10.4471/generos.2017.2667

\section{PLEASE SCROLL DOWN FOR ARTICLE}

The terms and conditions of use are related to the Open Journal System and to Creative Commons Attribution License (CC-BY). 
GÉNEROS-Multidisciplinary Journal of Gender Studies Vol. 6 No.2 June 2017 pp. 1385-1388

\section{Review}

Jiménez Cortés, R., Rebollo Catalán, A. y García Pérez, R. (Coords.) (2016). Aprendizaje con TIC para la Inclusión Digital. Las Mujeres como Tejedoras de las Redes Sociales. Madrid: Síntesis. ISBN: 978-84-9077-373-4

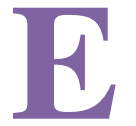

1 libro Aprendizaje con TIC para la inclusión digital. Las mujeres como tejedoras de las redes sociales es el fruto de una investigación cuya principal investigadora es Rocío Jiménez Cortés y coordinadores son Ángeles Rebollo Catalán y Rafael García Pérez. El conjunto de autores/as que han participado en este libro son: María José Rubio Hurtado, Anna Escofet Roig, Estrella Ruiz Pinto, Luisa Veja Caro, Virginia Mayor Buzón, Olga Buzón García, Alba Vico Bosh, Prudencia Gutiérrez Esteban, Sixto Cubo Delgado, Francisco de Paula Rodríguez Miranda, Rosario Rodríguez Díaz y María Lourdes Hernández Rincón.

Desde un enfoque psicológico y pedagógico, y a lo largo de 9 capítulos, se abordan preguntas en torno al aprendizaje con TIC para la inclusión digital en el colectivo femenino, para aportar una reflexión profunda de la situación actual entre redes sociales y mujeres.

Este libro resulta innovador porque nos muestra desde una perspectiva muy próxima, casi personal, la manera como aprenden y dinamizan sus propios procesos de auto-inclusión digital las mujeres adultas. En este sentido, aporta un conocimiento detallado del papel que están jugando las tecnologías digitales actuales en la vida de una amplia diversidad de mujeres.

Con esta investigación se pretende aumentar la presencia y la participación de las mujeres en las TIC, mejorar los contenidos digitales específicos que son de interés para ellas e incrementar la confianza y seguridad de las mujeres en entornos digitales que les ha sido robada por 
ejercer los supuestos roles destinados a ellas por la sociedad. Esto permite desarrollar diseños tecno-pedagógicos de aprendizaje formal, informal y no formal adecuados a sus necesidades y experiencias vitales. Por este motivo, resulta especialmente importante adaptar las metodologías de aprendizaje a los diferentes perfiles (desempleadas, emprendedoras, y del ámbito rural) en el uso de las tecnologías digitales y el desarrollo de competencias digitales.

La obra se divide en tres bloques. En este primer bloque los autores nos detallan las políticas de inclusión digital en clave de género, cómo superar la brecha de género y la alfabetización digital en las redes sociales con especial relevancia a la distinción entre mujeres consumidoras y prosumidoras de TIC.

El segundo bloque representa las voces y experiencias de una diversidad de mujeres. Desde una perspectiva social, nos lleva a conocer de primera mano los testimonios de 1.340 mujeres, su entorno familiar y el rol que desempeñan en él. Destacan el uso de Facebook como una herramienta polivalente.

El último bloque, aborda el aprendizaje en red, los entornos y ecosistemas digitales por los que discurren en el camino hacia el empoderamiento. Destacan dos puntos: la concepción de la mujer como una ciudadana de segundo orden y por lo cual los gobiernos no se sienten en la obligación de elaborar unas leyes que aborden la inclusión digital de las mujeres y, por otra parte, las formas de aprendizaje de las mujeres profesionales por la combinación de estrategias de aprendizaje que utilizan y por los diferentes medios en los que lo hacen.

En este libro encontramos mucho más que una mera descripción de las situaciones de aprendizaje con TIC. Encontramos un análisis detallado del día a día de estas mujeres, desde la gestión de su tiempo en base a las prioridades que su familia y entorno les demanda, la disponibilidad de horarios y de equipamientos y hasta las maneras en las que hacen uso de las TIC para no ser criticadas por maridos e hijos/as que éstas han experimentado. Los autores explican el proceso de aprendizaje de TIC en la mujer pero siempre vinculándola a su construcción como madre y esposa, así como a las necesidades materiales del mercado laboral en cada momento.

Por último, incorpora unos matices de clase social. Inevitablemente las mujeres emprendedoras y con lo cual, con mayores recursos económicos, nos muestran una historia diferente. Estas mujeres son respetadas, tienen 
1388 Cabanillas Sánchez - Aprendizaje con TIC para la Inclusión Social [Book Review]

todo el equipamiento que necesitan y acceden a las TIC desde cualquier lugar y a cualquier hora. Del mismo modo cuentan con diferentes entornos y redes de aprendizajes. Son mujeres que equilibran de la mejor manera posible, la vida personal con la profesional incluyendo la lucha constante contra el techo de cristal y el suelo pegajoso con el único objetivo de alcanzar su empoderamiento en una sociedad cambiante.

María José Cabanillas Sánchez, Universidad de Extremadura cabanillasmj@gmail.com 